OPEN ACCESS

Edited by:

Aurel Popa-Wagner,

University of Rostock, Germany

Reviewed by:

Douglas Watt,

Quincy Medical Center \& Cambridge

Health Alliance, USA

Raluca Sandu Vintilescu,

University of Medicine and Pharmacy

of Craiova, Romania

*Correspondence:

Mercè Pallàs

pallas@ub.edu

Received: 22 July 2016 Accepted: 29 September 2016

Published: 18 October 2016

Citation:

Griñan-Ferré C, Puigoriol-lllamola D, Palomera-Ávalos $V$,

Pérez-Cáceres $D$,

Companys-Alemany J, Camins A, Ortuño-Sahagún $D$, Rodrigo MT and

Pallàs M (2016) Environmental Enrichment Modified Epigenetic Mechanisms in SAMP8 Mouse Hippocampus by Reducing Oxidative

Stress and Inflammaging

and Achieving Neuroprotection.

Front. Aging Neurosci. 8:241.

doi: 10.3389/fnagi.2016.00241

\section{Environmental Enrichment Modified Epigenetic Mechanisms in SAMP8 Mouse Hippocampus by Reducing Oxidative Stress and Inflammaging and Achieving Neuroprotection}

\author{
Christian Griñan-Ferré1, Dolors Puigoriol-Illamola ${ }^{1}$, Verónica Palomera-Ávalos ${ }^{1}$, \\ David Pérez-Cáceres ${ }^{2}$, Júlia Companys-Alemany ${ }^{1}$, Antonio Camins ${ }^{1}$, \\ Daniel Ortuño-Sahagún ${ }^{3}, M$. Teresa Rodrigo ${ }^{2}$ and Mercè Pallàs ${ }^{1 *}$
}

\begin{abstract}
${ }^{1}$ Department of Pharmacology, Toxicology and Therapeutic Chemistry (Pharmacology Section) and Institute of Neuroscience, University of Barcelona, Barcelona, Spain, ${ }^{2}$ Animal Experimentation Unit, Faculty of Pharmacy, University of Barcelona, Barcelona, Spain, ${ }^{3}$ Instituto de Investigación en Ciencias Biomédicas, Centro Universitario de Ciencias de la Salud, Universidad de Guadalajara, Guadalajara, Mexico
\end{abstract}

With the increase in life expectancy, aging and age-related cognitive impairments are becoming one of the most important issues for human health. At the same time, it has been shown that epigenetic mechanisms are emerging as universally important factors in life expectancy. The Senescence Accelerated Mouse P8 (SAMP8) strain exhibits age-related deterioration evidenced in learning and memory abilities and is a useful model of neurodegenerative disease. In SAMP8, Environmental Enrichment (EE) increased DNA-methylation levels (5-mC) and reduced hydroxymethylation levels (5$\mathrm{hmC}$ ), as well as increased histone $\mathrm{H} 3$ and $\mathrm{H} 4$ acetylation levels. Likewise, we found changes in the hippocampal gene expression of some chromatin-modifying enzyme genes, such as Dnmt3b, Hdac1, Hdac2, Sirt2, and Sirt6. Subsequently, we assessed the effects of EE on neuroprotection-related transcription factors, such as the Nuclear regulatory factor 2 (Nrf2)-Antioxidant Response Element pathway and Nuclear Factor kappa Beta (NF-kB), which play critical roles in inflammation. We found that EE produces an increased expression of antioxidant genes, such as Hmox1, Aox1, and Cox2, and reduced the expression of inflammatory genes such as $I L-6$ and $C x C / 10$, all of this within the epigenetic context modified by EE. In conclusion, EE prevents epigenetic changes that promote or drive oxidative stress and inflammaging.

Keywords: neurodegeneration, environmental enrichment, oxidative stress, epigenetics, inflammation

\section{INTRODUCTION}

With the increase in the average life expectancy of the population worldwide, cognitive frailty is emerging as one of the most important issues of human health, with a rising percentage of the population affected by cognitive decline and dementia. However, it remains impossible to delineate the precise moment at which the brain begins to age (Fjell et al., 2014). 
Alzheimer's Disease (AD) is the most prevalent dementia and its progression is influenced by both genetic and environmental factors (Grupe et al., 2007; Avramopoulos, 2009; Harold et al., 2009). These gene-environment interactions may influence and trigger pathogenic pathways that determine the severity and progression of several degenerative diseases (Mastroeni et al., 2009; Bradley-Whitman and Lovell, 2013; Millan, 2014; GriñánFerré et al., 2015). In addition, a growing body of evidence demonstrated that the effects of environmental factors are mainly orchestrated by epigenetic mechanisms. In $\mathrm{AD}$, only a small percentage of the cases $(<5 \%)$ are consistent with inheritance. Even in the familial forms of $\mathrm{AD}$, penetrance is not complete and is also highly variable between individuals with the same genetic background (Mastroeni et al., 2009, 2011). Consequently, the effect of environmental factors is of remarkable importance. In this respect, epigenetic changes, or other forms of geneenvironment interaction, could be involved in determining the onset of $\mathrm{AD}$ and also aging, which is the most important risk factor for development of the pathology (López-Otín et al., 2013; Spiegel et al., 2014).

In particular, lines of evidence indicate that the methylation and hydroxymethylation state of the DNA plays an important role in controlling gene expression, and also learning and memory, including memory formation and maintenance of learning and memory decline during aging (Levenson et al., 2004; Miller and Sweatt, 2007; Liu et al., 2009; Day and Sweatt, 2010; Mikaelsson and Miller, 2011; Morris et al., 2014).

Similarly, epigenetic mechanisms, i.e., alterations of histone proteins and DNA, are essential for hippocampal synaptic plasticity and cognitive function disease (López-Otín et al., 2013). Additionally, recent works (Holliday, 2006; Fischer et al., 2007; Gräff and Tsai, 2013) demonstrate that chromatin regulation, and in particular histone acetylation, could be important for modulating the cognitive outcome of several neurodegenerative disorders. Moreover, exposure to an EE has been shown to have a protective effect in mouse models by slowing disease progression and reducing $\mathrm{AD}$-like cognitive impairment (Barak et al., 2013).

Although the mechanisms by which EE preserves cognition remain unknown, our work team recently demonstrated that $\mathrm{EE}$ is an experimental strategy to alleviate cognitive decline and neuroprotection in Senescence Accelerated Mice P8 (SAMP8) (Griñán-Ferré et al., 2015). Moreover, changes in modulators of epigenetic machinery, such as LSD1 or CoREST, were found changed after EE in this strain and were accompanied by cognitive feature improvements (Griñán-Ferré et al., 2015). These results correlated with the activation of endogenous neuroprotective pathways, thus implicating these in the beneficial effects of EE on cognition.

Many studies have demonstrated that EE changes promoter DNA methylation states while changing the expression of DNA MethylTransferases (DNMTs) (Christensen et al., 2009; Marsit et al., 2009; Madrigano et al., 2011; Barrès et al., 2012). Therefore, gene-environment interactions, mediated at the epigenetic level, may be an intermediary step in providing an appropriate organism response to changes in the environment (Irier et al., 2014).
There is evidence that EE attenuated Oxidative Stress (OS) through elevated Nrf2 levels in hippocampi (Snow et al., 2015). In addition, EE elicits anti-inflammatory effects through interaction with several immune signaling pathways, including Interleukin 6 (IL-6). Diminution in the proinflammatory process after EE might be due by changes in NF- $\mathrm{B}$ translocation to the nucleus, reducing chemokines (Costa et al., 2007; Jurgens and Johnson, 2012; Williamson et al., 2012).

The goal of the present work was to delve deep into the epigenetic mechanisms influenced by EE that rendered the previously described beneficial effects by preventing cognitive impairment and neuronal dysfunction. We focus on antioxidant and inflammaging processes modulated by EE exposure in SAMP8.

\section{MATERIALS AND METHODS}

\section{Animals and Enriched Environmental Experimental Design}

Male SAMP8 mice $(n=20)$ were used with free access to food and water, under standard temperature conditions $\left(22 \pm 2^{\circ} \mathrm{C}\right)$ and 12 h:12-h light-dark cycles (300 lux/0 lux).

The animals were maintained until day 21 with their mothers, and afterward were separated into cages at up to 3 months-of-age. Ten SAMP8 were employed for the Environmental Enrichment (EE) group (SAMP8 EE), and 10 were maintained under standard conditions as Control mice (SAMP8 Ct).

In the present study, we utilized the novel objects paradigm. Therefore, plastic tubes ( $20 \mathrm{~cm}$ long and $2.5 \mathrm{~cm}$ in diameter) were placed in EE cardboard-house cages, in addition to plastic dolls or toys, which were added, extracted, or changed each week.

Mice were treated according to European Community Council Directive 86/609/EEC and the procedures established by the Department d'Agricultura, Ramaderia i Pesca of the Generalitat de Catalunya, Spain. Every effort was made to minimize animal suffering and to reduce the number of animals.

\section{Behavioral and Cognitive Experiments Novel Object Recognition Test (NORT)}

The protocol employed was a modification of Ennaceur and Delacour (1988) and Ennaceur and Meliani (1992). In brief, mice were placed in a $90^{\circ}$-, two-arm, $25-\mathrm{cm}$-long, 20-cm-high, 5 -cm-wide black maze. The walls could be removed for easy cleaning. Light intensity in mid-field was 30 lux. The objects to be discriminated were made of plastic and were chosen not to frighten the mice, and objects with parts that could be bitten were avoided. Before performing the test, the mice were individually habituated to the apparatus for $10 \mathrm{~min}$ during 3 days. On day 4, the animals were submitted to a 10-min acquisition trial (first trial), during which they were placed in the maze in the presence of two identical, novel objects $(\mathrm{A}+\mathrm{A}$ or $\mathrm{B}+\mathrm{B})$ at the end of each arm. A 10-min retention trial (second trial) was carried out $2 \mathrm{~h}$ later. During this second trial, objects A and B were placed in the maze, and the behavior of the mice was recorded with a camera. Time that mice explored the New object (TN) and Time that mice explored the Old object (TO) were measured. A Discrimination 
Index (DI) was defined as (TN-TO)/(TN+TO). In order to avoid object preference biases, objects $\mathrm{A}$ and $\mathrm{B}$ were counterbalanced so that one half of the animals in each experimental group were first exposed to object $A$ and then to object $B$, whereas the other half first saw object $B$ and then object $A$. The maze and the objects were cleaned with $96^{\circ}$ ethanol after each test in order to eliminate olfactory cues.

\section{Morris Water Maze Test}

An open circular pool (100 cm in diameter, $50 \mathrm{~cm}$ in height) was filled halfway with water (Morris, 1981) and the temperature was maintained at $22^{\circ} \mathrm{C} \pm 1$. Two principal perpendicular axes were defined; thus, the water surface was divided into four quadrants (NE, SE, SW, and NW) and five starting points were set (NE, E, SE, S, and SW). Four visual clues were placed on the walls of the tank (N, E, S, and W). Non-toxic, white latex paint was added to make the water opaque, and a white escape platform was submerged $1 \mathrm{~cm}$ below the water level (approximately in the middle of one of the quadrants).

The animals' swimming paths were recorded by a video camera mounted above the center of the pool, and data were analyzed with SMART version 3.0 software. The learning phase consisted of 6 days of trials for each mouse. The animals were submitted to five trials each day starting from the positions set (in random order) and without a resting phase between each trial and the subsequent one. At each trial, the mouse was placed gently into the water, facing the wall of the pool, and allowed to swim for $60 \mathrm{~s}$. If not able to locate the platform in this period of time, the mouse was guided to the platform by the investigator. Animals were left on the platform each time for $30 \mathrm{~s}$ in order to allow for spatial orientation. A memory test was performed at the end of the learning days, in which the platform was removed and the time spent and the distance swum by each mouse in each quadrant was measured.

\section{Immunodetection Experiments}

\section{Brain Processing and Subcellular Fractionation}

After the behavioral test, the animals were killed by cervical dislocation. Afterward, the brains were immediately removed and the hippocampi were then isolated, frozen on powdered dry ice, and maintained at $-80^{\circ} \mathrm{C}$ until protein extraction.

For subcellular fractionation, $150 \mu \mathrm{L}$ of buffer A $(10 \mathrm{mM}$ HEPES pH 7.9, $10 \mathrm{mM} \mathrm{KCl,} \mathrm{0.1} \mathrm{mM} \mathrm{EDTA} \mathrm{pH} \mathrm{8,} 0.1 \mathrm{mM}$ EGTA pH 8, 1 mM DTT, 1 mM PMSF, protease inhibitors) were added to each sample and incubated on ice for $15 \mathrm{~min}$. After this time, the samples were homogenized with a tissue homogenizer, $12.5 \mu \mathrm{L}$ Igepal 1\% were added, and the Eppendorf was vortexed for $15 \mathrm{~s}$. Following $30 \mathrm{~s}$ of full-speed centrifugation at $4^{\circ} \mathrm{C}$, supernatants were collected (cytoplasmic fraction); $80 \mu \mathrm{L}$ of buffer C (20 mM HEPES pH 7.9, 0,4M NaCl, 1 mM EDTA pH 8, 0.1 mM EGTA pH 8, 20\% Glycerol $1 \mathrm{mM}$ DTT, $1 \mathrm{mM}$ PMSF, protease inhibitors) was added to each pellet and incubated under agitation at $4^{\circ} \mathrm{C}$ for $15 \mathrm{~min}$. Subsequently, samples were centrifuged for $10 \mathrm{~min}$ at full speed at $4^{\circ} \mathrm{C}$. Supernatants were collected (nuclear fraction) and $40 \mu \mathrm{L}$ of buffer $\mathrm{A}+\mathrm{HCl}$ (buffer A with 0.2 $\mathrm{N} \mathrm{HCl}$ ) was added to the pellet. After a 30-min incubation on ice, samples were centrifuged, again at full speed, at $4^{\circ} \mathrm{C}$ for $10 \mathrm{~min}$ and the supernatants were collected (the histone fraction).

\section{Western Blotting}

For the Western blot (wb) experiment, aliquots of homogenized hippocampus, $15 \mu \mathrm{g}$ protein for nuclear and cytoplasmic fractions, and $5 \mu \mathrm{g}$ for the histone fraction were used. The protein samples were separated by SDS-PAGE (8-18\%) and transferred onto PVDF membranes (Millipore). The membranes were blocked in 5\% non-fat milk in TBS containing $0.1 \%$ Tween 20 (TBS-T) for $1 \mathrm{~h}$ at room temperature, followed by an overnight incubation at $4^{\circ} \mathrm{C}$ with the primary antibodies listed in Table 1. Membranes were then washed and incubated with secondary antibodies for $1 \mathrm{~h}$ at room temperature. Immunoreactive protein was viewed with a chemiluminescencebased detection kit, following the manufacturer's protocol (ECL Kit; Millipore) and digital images were acquired using a ChemiDoc XRS+ System (BioRad). Semi-quantitative analyses were carried out utilizing Image Lab software (BioRad) and results were expressed in Arbitrary Units (AU). Protein loading was routinely monitored by phenol red staining of the membrane or by immunodetection of GADPH.

\section{Global DNA Methylation and Hydroxymethylation Quantification}

For global DNA, methylation and hydroxymethylation was performed according to the manufacturer's instructions, first using the FitAmpTM Blood and Cultured Cell DNA Extraction Kit, which is designed for rapid isolation of pure genomic DNA from a small amount of blood or mammalian cells. The second part was performed employing the Methylflash Methylated DNA Quantification Kit (Epigentek, Farmingdale, NY, USA) and the Methylflash Hydroxymethylated DNA Quantification Kit. Briefly, methylated DNA and hydroxymethymethylated DNA were detected using capture and detection antibodies to 5-mC and 5-hmC and then quantified colorimetrically by reading absorbance at $450 \mathrm{~nm}$ using the Microplate Photometer. The absolute amount of methylated or hydroxymethylated DNA (proportional to the Optical Density [OD] intensity) was measured and was quantified using a standard curve plotting the OD values vs. five serial dilutions of control methylated DNA (0.5-10 ng).

\section{RNA Extraction and Gene Expression Determination}

Total RNA isolation was carried out by means of Trizol reagent following the manufacturer's instructions. RNA content in the samples was measured at $260 \mathrm{~nm}$, and sample purity was determined by the A260/280 ratio in a NanoDrop ${ }^{\mathrm{TM}}$ ND-1000 (Thermo Scientific). Samples were also tested in an Agilent 2100B Bioanalyzer (Agilent Technologies) to determine the RNA integrity number. Reverse TranscriptionPolymerase Chain Reaction (RT-PCR) was performed as follows: $2 \mu \mathrm{g}$ of messenger RNA (mRNA) was reverse-transcribed using the High Capacity complementary DNA (cDNA) 
TABLE 1 | Antibodies used in Western blot studies.

\begin{tabular}{llll}
\hline Antibody & Host & Source/Catalog & WB dilution \\
\hline p65 & Rabbit & Cell signaling/D14E12 & $1: 1000$ \\
NRF2 & Rabbit & Cell signaling/DIZ9C & $1: 1000$ \\
Acetyl Histone H3 & Rabbit & Millipore/06-599 & $1: 1000$ \\
Acetyl Histone H4 & Sheep & R\&D systems/AF5215 & $1: 1000$ \\
GAPDH & Mouse & Millipore/MAB374 & $1: 2000$ \\
Histone H3 & Rabbit & Santa Cruz & $1: 500$ \\
& & Biotech/sc-10809 & \\
Histone H4 & Mouse & Cell signaling/L64Cl & $1: 1000$ \\
TBP & Mouse & Abcam/51841 & $1: 1000$ \\
$\begin{array}{l}\text { Donkey-anti-goat HRP } \\
\text { conjugated }\end{array}$ & & Santa Cruz Biotech/ & $1: 3000$ \\
$\begin{array}{l}\text { Goat-anti-mouse HRP } \\
\text { conjugated }\end{array}$ & & Sc-2020 & $1: 2000$ \\
$\begin{array}{l}\text { Rabbit-anti-sheep HRP } \\
\text { conjugated }\end{array}$ & & Biorad/\# 170-5047 & $1: 2000$ \\
$\begin{array}{l}\text { Goat-anti-rabbit HRP } \\
\text { conjugated }\end{array}$ & & Abcam/ab97130 & $1: 2000$ \\
\hline
\end{tabular}

Reverse Transcription Kit (Applied Biosystems). Real-time quantitative PCR (qPCR) was employed to quantify the mRNA expression of a set of chromatin- modifying enzyme genes, including Dnmt1, Dnmt3a, Dnmt3b, methylcytosine dioxygenase TET1 (Tet1), methylcytosine dioxygenase TET2 (Tet2), Sirtuin 1 (Sirt1), Sirtuin 2 (Sirt2), Sirtuin 6 (Sirt6), Histone deacetylase 1 (Hdac1), Histone deacetylase 2 (Hdac2), OS genes Heme oxygenase (decycling) 1 (Hmox 1 ), Aldehyde oxidase 1 (Aox1), Aldehyde dehydrogenase 2 (Aldh2),
Cyclooxygenase 2 (Cox2), inflammatory genes Interleukin 6 (IL-6), C-X-C motif chemokine 10 (Cxcl10), and Tumor necrosis factor alpha $(\operatorname{Tn} f-\alpha)$. Normalization of expression levels was performed with actin for SYBER Green and TATA-binding protein $(T b p)$ for TaqMan. The primers were those listed in Table 2.

For SYBER Green, real-time PCR was performed in the Step One Plus Detection System (Applied Biosystems) employing the SYBR Green PCR Master Mix (Applied Biosystems). Each reaction mixture contained $7.5 \mu \mathrm{L}$ of $\mathrm{CDNA}$, whose concentration was $2 \mu \mathrm{g}, 0.75 \mathrm{uL}$ of each primer (whose concentration was $100 \mathrm{nM}$ ), and $7.5 \mu \mathrm{L}$ of SYBR Green PCR Master Mix (2X), and for TaqMan gene expression assays (Applied Biosystems), for each $20 \mu \mathrm{L}$ of TaqMan reaction, $9 \mu \mathrm{L}$ cDNA (18 ng) was mixed with $1 \mu \mathrm{L} 20 \mathrm{X}$ probe of TaqMan Gene Expression Assays and $10 \mu \mathrm{L}$ of $2 \mathrm{X}$ TaqMan Universal PCR Master Mix.

Data were analyzed utilizing the comparative Cycle threshold (Ct) method ( $\Delta \Delta \mathrm{Ct})$, where the actin transcript level was used to normalize differences in sample loading and preparation. Each sample ( $n=4-5)$ was analyzed in triplicate, and results represented the $n$-fold difference of transcript levels among different samples.

\section{Data Analysis}

Data are expressed as the mean \pm Standard Error of the Mean (SEM) from at least 5-6 samples. Data analysis was conducted using GraphPad Prism version 6 statistical software. Means were compared with the two-tailed, unpaired Student $t$-test. Statistical significance was considered when $p$-values were $<0.05$.

\section{TABLE 2 | Primers and probes used in qPCR studies.}

\begin{tabular}{|c|c|c|c|}
\hline Target & $\begin{array}{l}\text { Product } \\
\text { size (bp) }\end{array}$ & Forward primer $\left(5^{\prime}-3^{\prime}\right)$ & Reverse primer $\left(5^{\prime}-3^{\prime}\right)$ \\
\hline \multicolumn{4}{|c|}{ SYBR Green primers } \\
\hline Dnmt3b & 142 & TGCCAGACCTTGGAAACCTC & GCTGGCACCCTCTTCTTCAT \\
\hline Tet1 & 188 & CTGCCAACTACCCCAAACTCA & TCGGGGTITGTCTTCCGTT \\
\hline Tet2 & 113 & CCATCATGTTGTGGGACGGA & ATTCTGAGAACAGCGACGGT \\
\hline Sirt1 & 229 & AACACACACACAAAATCCAGCA & TGCAACCTGCTCCAAGGTAT \\
\hline Sirt2 & 248 & TGCAGGAGGCTCAGGATTC & GTCACTCCTTCGAGGGTCAG \\
\hline Sirt6 & 150 & GTCTCACTGTGTCCCTTGTCC & GCGGGTGTGATTGGTAGAGA \\
\hline Aox1 & 286 & CATAGGCGGCCAGGAACATT & TCCTCGTTCCAGAATGCAGC \\
\hline Cox2 & 126 & TGACCCCCAAGGCTCAAATA & CCCAGGTCCTCGCTTATGATC \\
\hline
\end{tabular}

Taqman probes

Dnmt3a

Hmox1

Tbp

$\begin{array}{ll}58 & \text { Mm00432881 m1 } \\ 69 & \text { Mm00516005_m1 } \\ 93 & \text { Mm00446971_m1 }\end{array}$




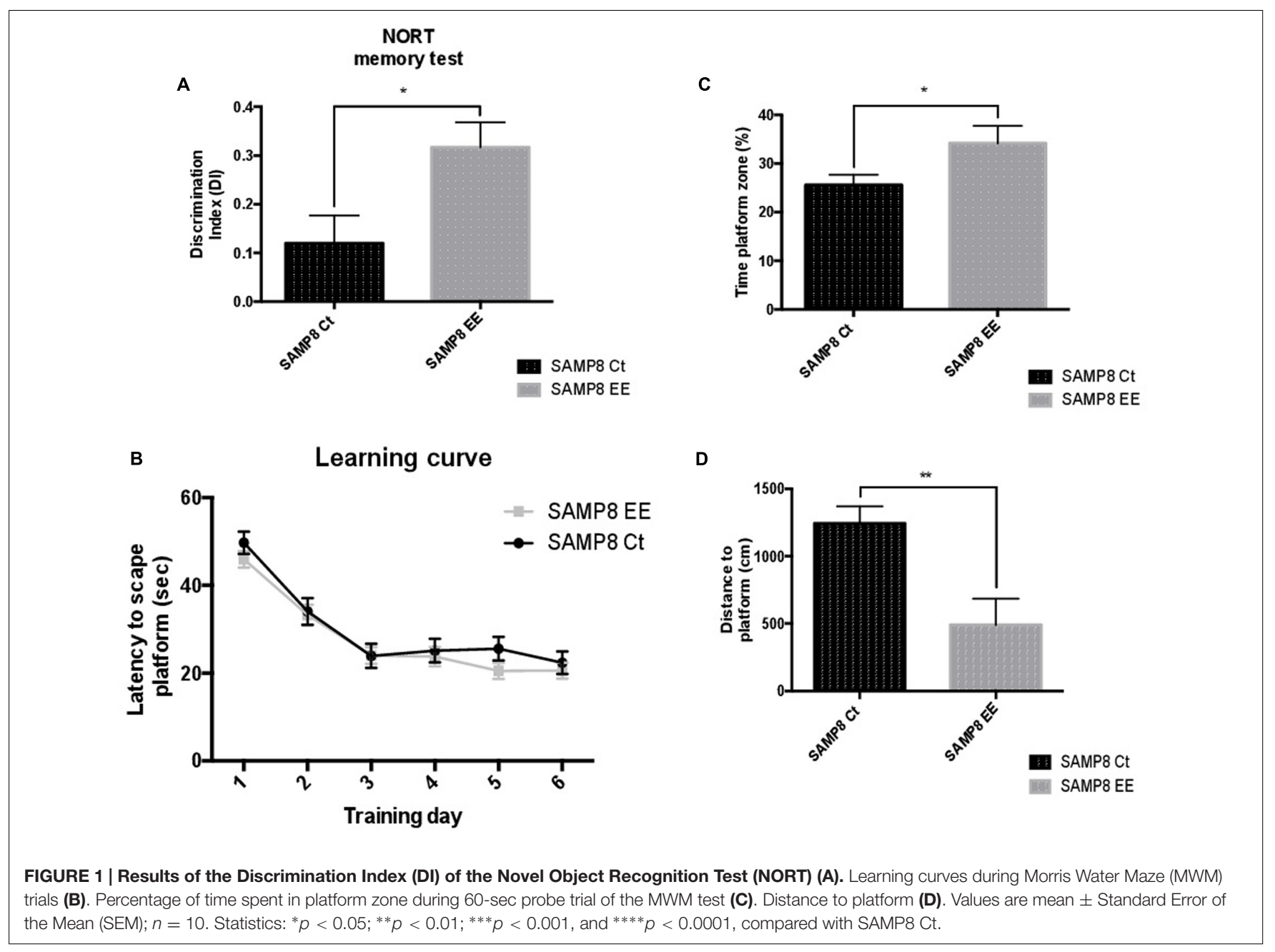

\section{RESULTS}

\section{EE Mitigates Cognitive Decline in Male SAMP8 Mice}

The Novel Object Recognition Test (NORT) demonstrated significant memory amelioration in SAMP8 EE mice in reference to SAMP8 Controls (Ct) (Figure 1A). Improvement in cognition was also demonstrated by the results obtained in the Morris Water Maze (MWM) test (Figure 1B). Moreover, time spent on platform quadrant was longer (Figure 1C); in contrast, distance swum to platform was significantly lesser in SAMP8 EE than in SAMP8 Ct (Figure 1D). These results corroborate that previously obtained, but with higher number of animals (Griñán-Ferré et al., 2015).

\section{Hippocampal Global Changes in DNA Methylation and Hydroxymethylation and Its Machinery after EE}

To address the question of whether or not EE could affect DNA methylation or hydroxymethylation, we determined 5-methylcytosine levels and found a significant increase in
SAMP8 EE in comparison with SAMP8 Ct (Figure 2A). In a parallel manner, 5-hmC levels were reduced in SAMP8 EE. When gene expression in DNMTs was studied, only significant decreases in Dnmt3b were found (Figure 2B). Moreover, Ten-eleven Translocation Methylcytosine Dioxygenase 1 (Tet1), but not Tet2, increased in SAMP8 EE mice (Figure 2B).

\section{Hippocampal Global Changes in Histone Acetylation Levels and Its Machinery after EE}

The main Histone DeACetylase (HDAC) members of families I and III were studied. HDAC 1 and 2 (representative of the HDAC class I family) exhibited a contrasting profile, with increased gene expression in $\mathrm{Hdacl}$ and a decrease in Hdac2. Sirt2 and Sirt6, members of HDAC family III, are increased after EE in SAMP8 (Figure 3C). Protein Acetylated H4 was significantly increased in SAMP8 EE, whereas Acetylated $\mathrm{H} 3$ showed increased levels that did not reach significance (Figures 3A,B), demonstrating that higher levels of Hdac2 were determining in histone acetylation levels under the EE strategy in SAMP8. 
A

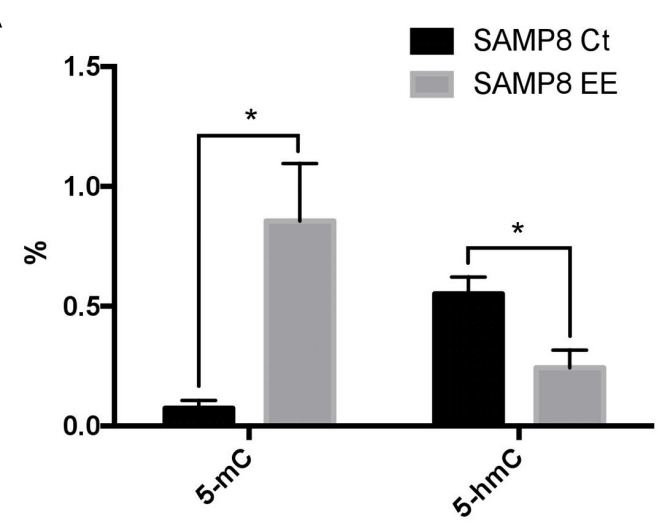

SAMP8 Ct
SAMP8 EE

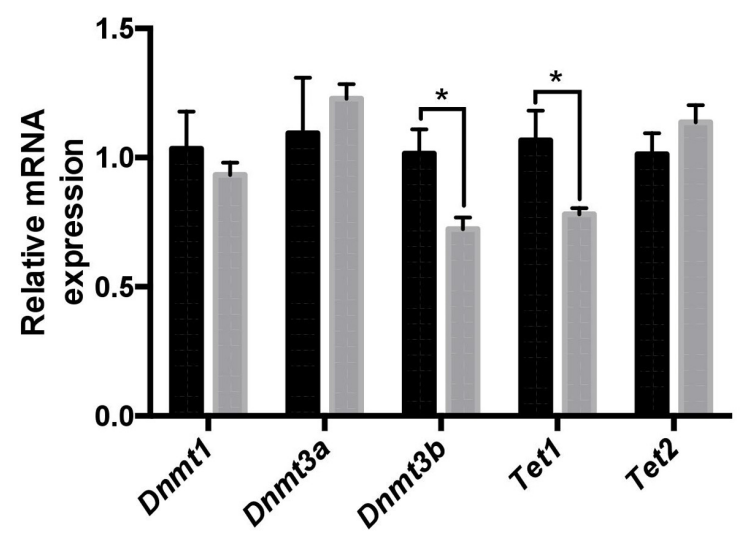

FIGURE 2 | Global 5-methylated cytosine and 5-hydroxymethylated cytosine levels (A). Gene expression for Dnmt1, Dnmt3a, Dnmt3b, Tet1, and Tet2 (B). Gene expression levels were determined by real-time PCR. Presented here are Mean \pm Standard Values are means (Standard Error of the Mean [SEM]) of the five independent experiments performed in triplicate. ${ }^{*} p<0.05 ;{ }^{* *} p<0.01{ }^{* * *} p<0.001$, and ${ }^{* * * *} p<0.0001$ compared with SAMP8 Ct.

\section{Molecular Changes in Nrf2 and NF-kB Pathways Induced by EE}

To address the question of whether EE increase in antioxidant enzymes is achieved by upregulating Nrf2, we studied protein levels and demonstrated that this transcription factor is translocated into the nucleus in the hippocampus of SAMP8 EE (Figures 4A,B). In parallel fashion, expression of Nrf2 target genes was significantly increased in these animals. Concretely, Hmox1, Aox1, and Cox2, genes coding for oxidative machinery proteins, were upregulated in SAMP8 EE compared with SAMP8 Ct (Figure 4C).

Previous results from our laboratory demonstrated that $I L$ 6 expression was lowered by EE in SAMP8. NF- $\mathrm{B}$ is a transcription factor related with inflammatory response and a master commander in the expression of proinflammatory genes. We found that EE reduced translocation to the nucleus (Figures 5A,B) and, most importantly, demonstrated that this reduction in nuclear translocation rendered a significantly decrease in $I L-6$ and $C x c l 10$ gene expression, although no changes were observed in $\operatorname{Tnf}-\alpha$ (Figure 5C).

\section{DISCUSSION}

In the present study, we showed that EE affected epigenetic markers and produced changes in the transcriptional control of oxidative and inflammaging markers in SAMP8. We hypothesize that changing the environment protectively would modulates a large constellation or at least a large subset of cellular phenotypes of aging. SAMP8 comprises a well-enough, but still not fully characterized, model for studying brain aging and neurodegeneration (Takeda, 2009; Pallàs, 2012). Experimental studies on SAMP8 postulated it as a non-transgenic murine model for late-onset AD (Pallàs et al., 2008; Morley et al., 2012b; Pallàs, 2012; Cheng et al., 2014), in addition to being a spontaneous senescence-accelerated mouse strain. These mice exhibited cognitive and emotional disturbances from young ages, probably due to early development of brain pathological hallmarks, such as OS, inflammation, and activation of neuronal death pathways, which mainly affects cerebral cortex and hippocampus (Canudas et al., 2005; Sureda et al., 2006; Cristòfol et al., 2012; Morley et al., 2012a). We have revealed the first, to our knowledge, clue regarding epigenetic modulation in inflammation and oxidative processes in SAMP8 (Alvarez-López et al., 2013) and in a model of familial AD (Griñán-Ferré et al., 2016). Therefore, an adequate epigenetic intervention during early life would be able to have influence on the dysfunctional or harmful molecular mechanisms affected in such a way that this would be able to exert a positive influence (Cosín-Tomás et al., 2014).

Molecular changes in young-age SAMP8 are based on cerebral and cognitive dysfunction (Yuan et al., 2012; AlvarezLópez et al., 2013), and these harmful changes were partially reverted in SAMP8 after EE early exposure, including cognitive, psychoemotional, and neurodegenerative hallmarks of $\mathrm{AD}$ (Griñán-Ferré et al., 2015). In addition to neuroprotection, EE was able to induce some type of epigenetic modulation in this strain, due to changes in CoREST and LSD1, in both hippocampus and cortex. Therefore, to open the focus on and to probe this epigenetic control, we demonstrated here that the DNA transcriptional control of specific genes indicated its being the initial and key step in the molecular stream developed in SAMP8 by EE, which improves cognition and ameliorates harmful factors gated to neurodegeneration, such as neuronal loss and apoptosis prevention, downregulation of kinase activity, and reduction in OS.

DNA methylation influences hippocampal memory formation, and growing evidence suggests that the regulation of epigenetic processes by EE, stress, and/or hormones can participate in memory function. In the present work and in accordance with the decrease in LSD1 transcription described in Griñán-Ferré et al. (2015), a higher degree of DNA hypermethylation was found in SAMP8 EE hippocampus. Conversely, only DNMT3b was reduced, whereas other DNMT 
A

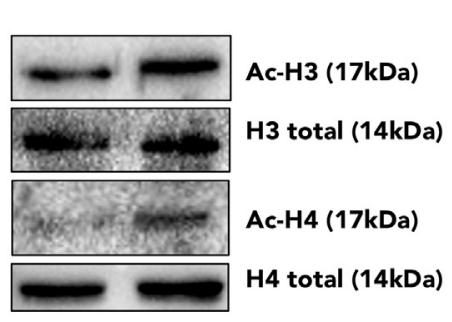

B

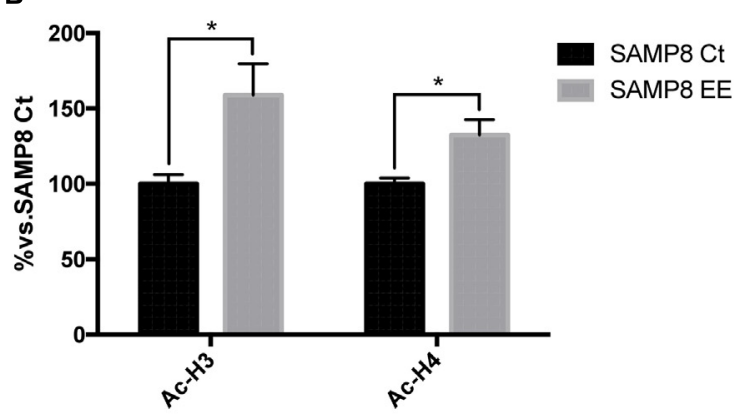

C

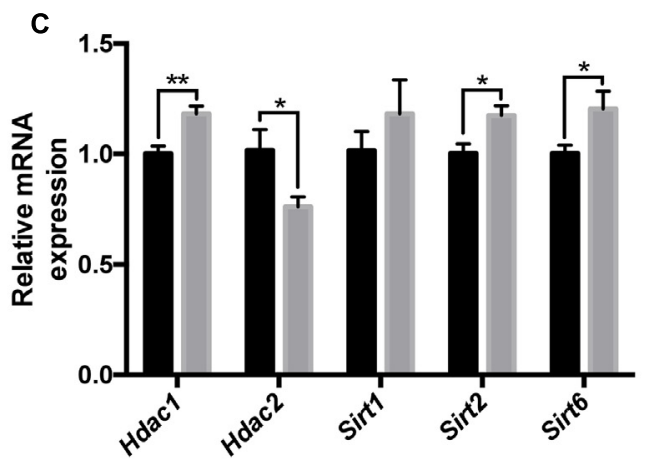

SAMP8 Ct SAMP8 EE

FIGURE 3 | Representative Western blot (wb) for acetylated and total H3 and H4 protein levels (A) and quantification after Enhanced Environment (EE) intervention (B). Deacetylase gene expression related with memory for Hdac1, Hdac2, Sirt1, Sirt2, and Sirt6 (C). Values in bar graphs are adjusted to 100\% for protein levels of Control SAMP8 (SAMP8 Ct). Gene expression levels were determined by real-time PCR. Mean \pm Standard Error of the Mean (SEM) from five independent experiments performed in duplicate are represented. ${ }^{*} p<0.05$ vs. Control SAMP8 (SAMP8 Ct).

were not modified. Usually, 5-mC decreases transcriptional access to DNA, although the functional effects of this gene silencing depend on the genes that are altered (AlvarezLópez et al., 2013). However, recent data suggest a more complex role for 5-mC, which also includes stimulation of gene expression (Weber et al., 2007; Wu and Zhang, 2010). Both, increases and decreases of $5-\mathrm{mC}$ have been described during aging (Richardson, 2003). Furthermore, pathological DNMT activity and aberrant 5-mC formation have been linked with neurodegeneration and apoptotic neuronal death (Chestnut et al., 2011; Hernandez et al., 2011). In these latter studies, neuroprotection was provided by DNMT inhibitors.

5-hmC is another epigenetic marker, catalyzed by the TET family, that appears to be particularly susceptible to developmental and aging-associated modifications (Flax and Soloway, 2011; Ito et al., 2011). According with other studies, diminution in 5-hmC, which paralleled a decrease in the Tet1 expression level, was found after EE in SAMP8. For instance, Irier et al. (2014) found that aged mice exposed to EE improved learning and memory, reducing 5-hmC-abundant hippocampus. However, in this work, no changes in Tet 1 expression were found, and differences can be explained by different timing in EE and also because of differences in strain and age. In sum, aging-associated 5-hmC alterations appear to be likely participants in the neuroplasticity of the aging brain.

In addition to DNA methylation changes, histone alterations are one of epigenetic mechanisms that are essential for hippocampal synaptic plasticity and cognitive function diseases (Zhao et al., 2012). In total, histone acetylation increases gene transcriptional activity by relaxing chromatin (de Ruijter et al., 2003). The role of histone acetylation in memory has been intensively explored in recent years. Increasing histone $\mathrm{H} 3$ and $\mathrm{H} 4$ acetylation by means of HDAC inhibitors in the dorsal hippocampus enhances several types of hippocampal-dependent memories (Stefanko et al., 2009; Haettig et al., 2011), and also rescued hippocampal memory deficits in mouse models of $\mathrm{AD}$ (Ricobaraza et al., 2009; Kilgore et al., 2010) and on aged rats (Neidl et al., 2016). Different classes of HDAC can be distinctly involved in memory formation or in neurodegeneration. For instance, Hdacl expression is upregulated in a mouse model for Huntington's disease (Quinti et al., 2010). However, mice lacking or overexpressing neuronal HDAC1 from early developmental stages are viable and display normal memory function (Guan et al., 2009). Here, a significant increase in $\mathrm{H} 3$ and $\mathrm{H} 4$ acetylation levels were found after EE in SAMP8. Although changes in Hdac1, Sirt2, and Sirt6 revealed an increase in gene expression, Hdac2 gene expression was significantly reduced, pointing to a major role of this deacetylase in the beneficial action of EE 
A

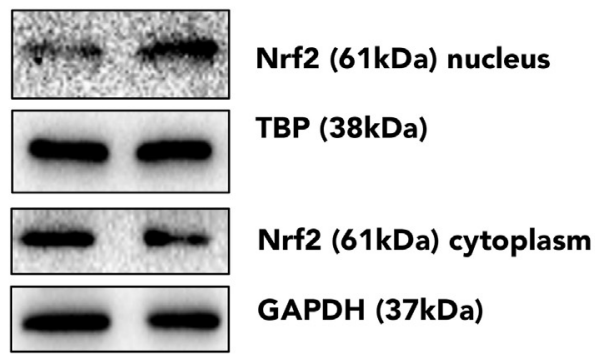

B

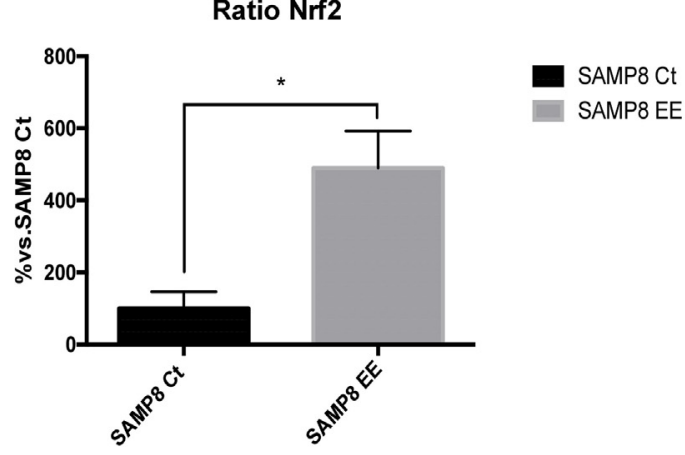

Nrf2 target genes

C

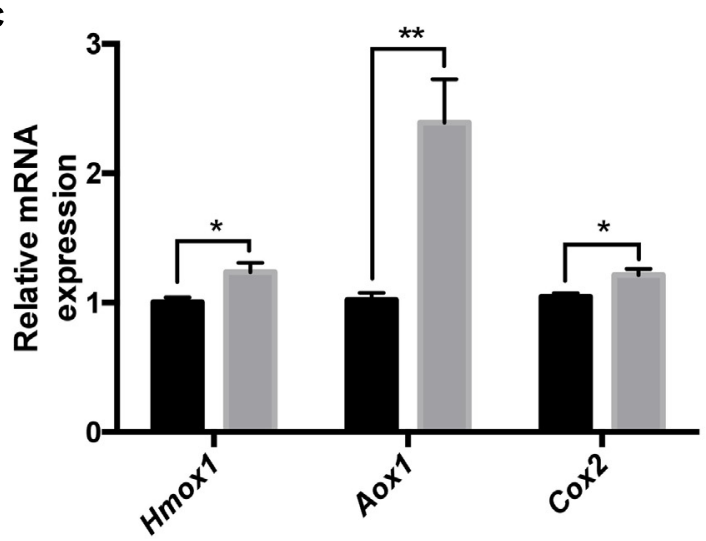

SAMP8 Ct SAMP8 EE

FIGURE 4 | Representative wb for Nuclear regulatory factor 2 (Nrf2) in cytoplasm and nucleus protein levels (A) and ration quantification (B). Nrf2 target-gene expression Hmox1, Aox1, and Cox2 (C). Values in bar graphs are adjusted to 100\% for protein levels of Control SAMP8 (SAMP8 Ct). For gene expression, levels were determined by real-time PCR. Mean \pm Standard Error of the Mean (SEM) from five independent experiments performed in duplicate are represented. $* p<0.05$ vs. Control SAMP8 (SAMP8 Ct).

on the SAMP8 strain (Griñán-Ferré et al., 2015). Interestingly, HDAC2 is one of the major regulators involved in the regulation of long-term memory. Specifically, mice overexpressing HDAC2 exhibited memory impairment, while HDAC2-knockout mice demonstrated enhanced synaptic plasticity (Guan et al., 2009).

On the other hand, and in the same line of all previously mentioned herein, we found an increase in two transcriptional regulatory pathways; Nrf2 and NF- $\kappa \mathrm{B}$, despite the hypermethylation induced by EE. Thus, we can deduce that EE-induced hypermethylation of low-density CpG islands were the promoter for Nrf2, the Antioxidant Response Element (ARE), and for NF- $\mathrm{BB}$.

It is well known that OS possesses a pre-eminent role in the pathogenesis of $\mathrm{AD}$, and in addition, decreased expression of Nrf2 has been reported in AD (Cao et al., 2015). In response to OS, Nrf2 is translocated from the cytoplasm to the nucleus and subsequently binds with ARE, in turn promoting the expression of a variety of OS-related genes (Alam et al., 1999).

We found that in SAMP8 EE, there was significant Nrf2 nuclear translocation, and this in turn could induce the transcription of Heme oxygenase (decycling) 1 (Hmox1),
Aldehyde oxidase 1 (Aox1), and Cyclooxygenase 2 (Cox2) genes. Hmox1 can exert cytoprotective effects against OS, whereas Aox 1 and Cox2 mediated Reactive Oxygen Species (ROS). These latter factors can act as hormetic regulators in an oxidative environment, favoring the cellular defenses confronting OS (Cai et al., 2012; Maeda et al., 2012). Other target genes, such as Aldehyde dehydrogenase 2 (Aldh2), were not affected (data not shown). It is also relevant that memory improvement in Nrf2level modulation has been reported in aged APP/PS1 mice (Kanninen et al., 2009) and in memory-impaired rats (Dwivedi et al., 2013).

On the other hand, the high levels of inflammatory mediators can increase a physiological response to certain pathological conditions in the nervous system, as $\mathrm{AD}$ or stroke, and It has been suggested that modulation of the signaling pathways of these mediators could be a potential therapeutic approach (Buga et al., 2013; Carriba and Comella, 2015). Here, we have studied NF- $\kappa \mathrm{B}$, that is a transcription factor in positive feedback with and exercising a regulatory role in relationship to classic pro-inflammatory signals that also impacts cell survival and apoptosis, allowing cells to adapt and respond to environmental 
A

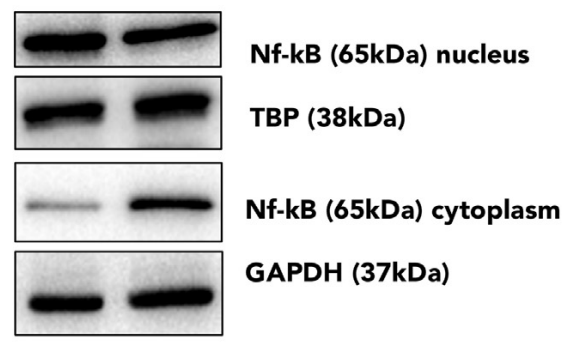

B

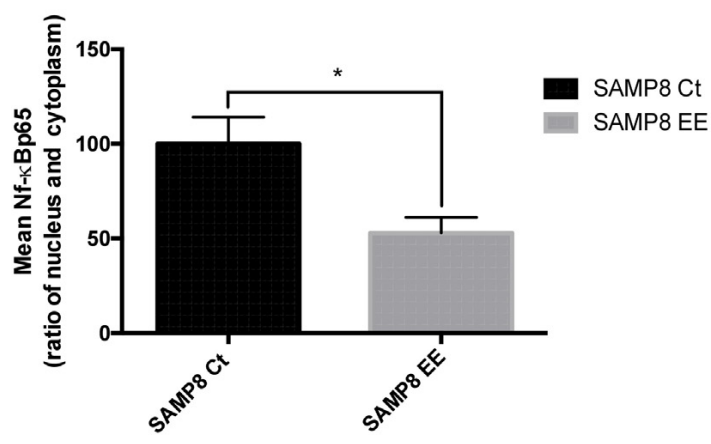

C Nf-kB target genes

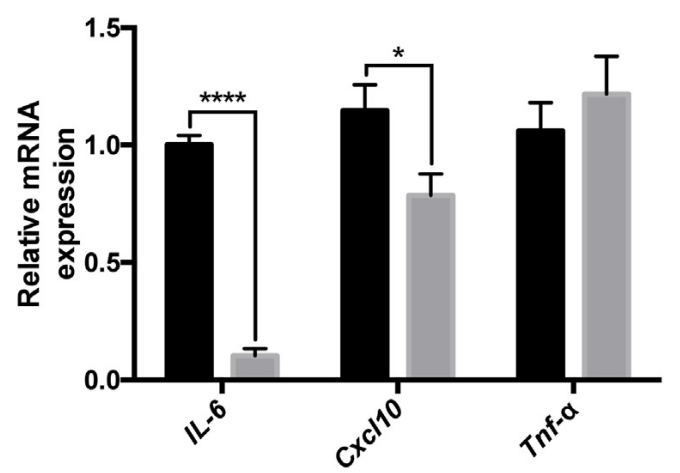

SAMP8 Ct

SAMP8 EE

FIGURE 5 | Representative wb for Nuclear Factor kappa Beta (NF- $\mathrm{kB}$ ) in cytoplasm and nucleus protein levels (A) and ration quantification (B). NF- $\mathrm{kB}$ target-gene expression IL-6, CxC/10, and Tnf- $\alpha$ (C). Values in bar graphs are adjusted to 100\% for protein levels of Control SAMP8 (SAMP8 Ct). For gene expression, levels were determined by real-time PCR. Mean \pm Standard Error of the Mean (SEM) from five independent experiments performed in duplicate are represented. ${ }^{*} p<0.05$ vs. Control SAMP8 (SAMP8 Ct).

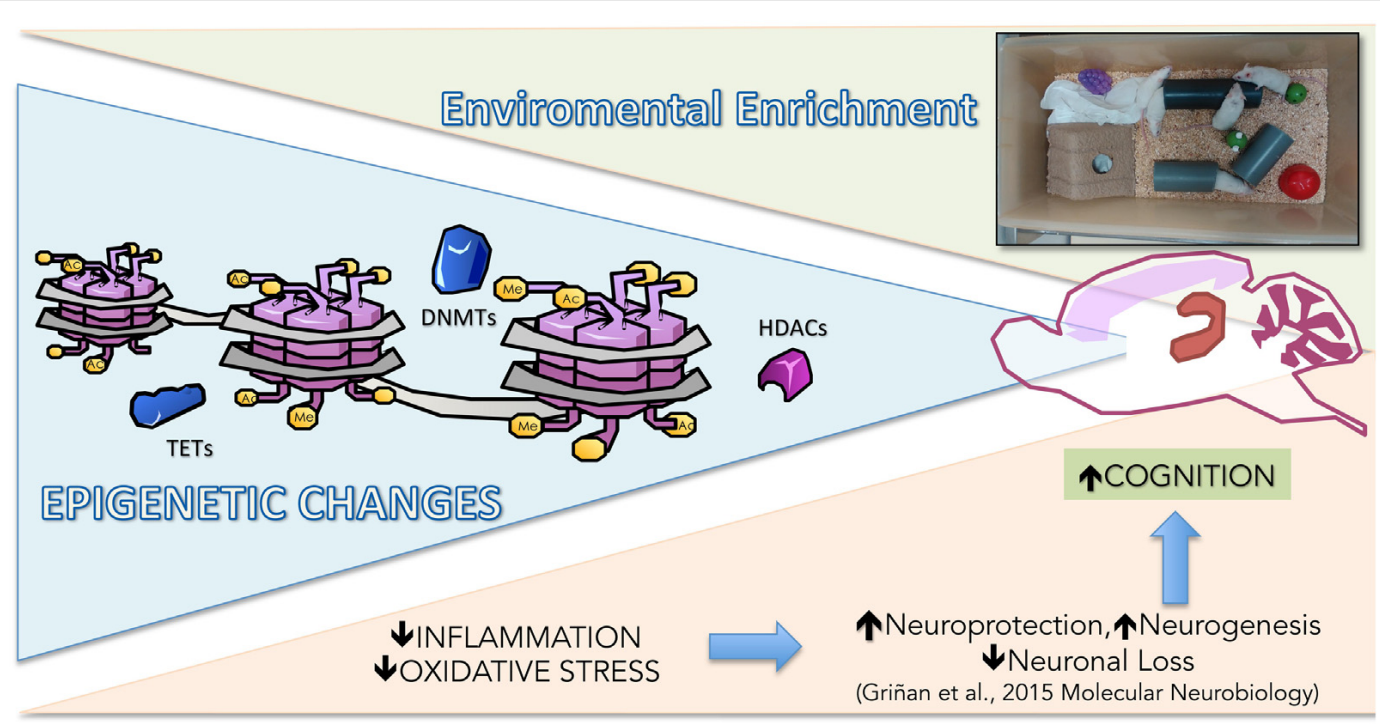

FIGURE 6 | Representative rendering of epigenetic and molecular mechanisms modified in Senescence-Accelerated Prone Mice P8 (SAMP8) after EE. 
changes (Oeckinghaus and Ghosh, 2009). In a previous work, we described that EE induced a significant decrease of $I L-6$ gene expression in the SAMP8 EE group compared with SAMP8 Ct (Griñán-Ferré et al., 2015). IL-6 is a pleiotropic inflammatory cytokine secreted by activated glia in the Central Nervous System (CNS) and is involved in mood disorders such as depression, also in aging process and the pathogenesis of neurodegenerative diseases such as AD (Quintanilla et al., 2004; Popa-Wagner et al., 2014). Our results demonstrate significant lowest genetic expression for $I L-6$, and also for CXcl10, but not for Tumor necrosis factor alpha $(T n f-\alpha)$ in the SAMP8 EE group. C-X$\mathrm{C}$ motif chemokine 10 ( $\mathrm{Cxcl10})$ CXCL10 is secreted by several cell types in response to InterFeroN gamma (IFN- $\gamma$ ) and was found increased in AD mouse models (Zaheer et al., 2013). These results coincide with a decrease in the nuclear translocation of proinflammatory transcription factor NF- $\kappa$ B in SAMP8 mouse EE.

Taken together, our results showed that crucial epigenetic modifications were demonstrated in the SAMP8 EE paradigm, reducing proinflammatory and OS pathways. Therefore, epigenetics orchestrated the homeostasis among DNA methylation, histone acetylation, and transcription factors, acting as a crosstalk mechanism and leading cognitive improvement and neuroprotection after EE in SAMP8 (Figure 6).

\section{REFERENCES}

Alam, J., Stewart, D., Touchard, C., Boinapally, S., Choi, A. M., and Cook, J. L. (1999). Nrf2, a Cap'n'Collar transcription factor, regulates induction of the heme oxygenase-1 gene. J. Biol. Chem. 274, 26071-26078. doi: $10.1074 /$ jbc.274.37.26071

Alvarez-López, M. J., Castro-Freire, M., Cosín-Tomás, M., Sanchez-Roige, S., Lalanza, J. F., Del, Valle J, et al. (2013). Long-term exercise modulates hippocampal gene expression in senescent female mice. J. Alzheimers Dis. 33, 1177-1190. doi: 10.3233/JAD-121264

Avramopoulos, D. (2009). Genetics of Alzheimer's disease: recent advances. Genome Med. 1, 34. doi: 10.1186/gm34

Barak, B., Shvarts-Serebro, I., Modai, S., Gilam, A., Okun, E., Michaelson, D. M., et al. (2013). Opposing actions of environmental enrichment and Alzheimer's disease on the expression of hippocampal microRNAs in mouse models. Transl. Psychiatry 3, e304. doi: 10.1038/tp.2013.77

Barrès, R., Yan, J., Egan, B., Treebak, J. T., Rasmussen, M., Fritz, T., et al. (2012). Acute exercise remodels promoter methylation in human skeletal muscle. Cell Metab. 15, 405-411. doi: 10.1016/j.cmet.2012. 01.001

Bradley-Whitman, M. A., and Lovell, M. A. (2013). Epigenetic changes in the progression of Alzheimer's disease. Mech. Ageing Dev. 134, 486-495. doi: 10.1016/j.mad.2013.08.005

Buga, A. M., Di Napoli, M., and Popa-Wagner, A. (2013). Preclinical models of stroke in aged animals with or without comorbidities: role of neuroinflammation. Biogerontology 14, 651-662. doi: 10.1007/s10522-0139465-0

Cai, C., Teng, L., Vu, D., He, J. Q., Guo, Y., Li, Q., et al. (2012). The heme oxygenase 1 inducer (CoPP) protects human cardiac stem cells against apoptosis through activation of the extracellular signal-regulated kinase (ERK)/NRF2 signaling pathway and cytokine release. J. Biol. Chem. 287, 33720-33732. doi: 10.1074/jbc.M112.385542

Canudas, A. M., Gutierrez-Cuesta, J., Rodríguez, M. I., Acuña-Castroviejo, D., Sureda, F. X., Camins, A., et al. (2005). Hyperphosphorylation of microtubule-associated protein tau in senescence-accelerated mouse (SAM). Mech. Ageing Dev. 126, 1300-1304. doi: 10.1016/j.mad.2005. 07.008

\section{AUTHOR CONTRIBUTIONS}

CG-F, DP-I, VP-A, DP-C, and JC-A performed the experiments. CG-F, MTR, and DP-C carried out the experimental intervention. CG-F and VP-A performed behavior experiments. DP-I and JC-A performed ELISA, and western blot analysis. CG-F and DP-I performed the RT-PCR experiments. CG-F, AC, and DO-S analyzed the data and drafted the manuscript. MP designed the experiments and supervised the study. All authors read and approved the final manuscript. All authors read and approved the final manuscript.

\section{FUNDING}

This study was supported by Spanish MINECO, and the European Regional Development Fund (SAF-2016-77703). CG-F, VP-A, AC, and MP belong to CIBERNED and 2014SGR 525.

\section{ACKNOWLEDGMENT}

We thank Maggie Brunner, M.A., for reviewing the language and style of the manuscript.

Cao, H., Wang, L., Chen, B., Zheng, P., He, Y., Ding, Y., et al. (2015). DNA demethylation upregulated Nrf2 expression in Alzheimer's disease cellular model. Front. Aging Neurosci. 7:244. doi: 10.3389/fnagi.2015.00244

Carriba, P., and Comella, J. X. (2015). Neurodegeneration and neuroinflammation: two processes, one target. Neural Regen. Res. 10, 1581-1583. doi: 10.4103/16735374.165208

Cheng, X., Zhou, W., and Zhang, Y. (2014). The behavioral, pathological and therapeutic features of the senescence-accelerated mouse prone 8 strain as an Alzheimer's disease animal model. Ageing Res. Rev. 13, 13-37. doi: 10.1016/j.arr.2013.10.002

Chestnut, B. A., Chang, Q., Price, A., Lesuisse, C., Wong, M., and Martin, L. J. (2011). Epigenetic regulation of motor neuron cell death through DNA methylation. J. Neurosci. 31, 16619-16636. doi: 10.1523/JNEUROSCI.163911.2011

Christensen, B. C., Houseman, E. A., Marsit, C. J., Zheng, S., Wrensch, M. R., Wiemels, J. L., et al. (2009). Aging and environmental exposures alter tissuespecific DNA methylation dependent upon CpG island context. PLoS Genet. 5:e1000602. doi: 10.1371/journal.pgen.1000602

Cosín-Tomás, M., Alvarez-López, M. J., Sanchez-Roige, S., Lalanza, J. F., Bayod, S., Sanfeliu, C., et al. (2014). Epigenetic alterations in hippocampus of SAMP8 senescent mice and modulation by voluntary physical exercise. Front. Aging Neurosci. 6:51. doi: 10.3389/fnagi.2014.00051

Costa, D. A., Cracchiolo, J. R., Bachstetter, A. D., Hughes, T. F., Bales, K. R., Paul, S. M., et al. (2007). Enrichment improves cognition in AD mice by amyloid-related and unrelated mechanisms. Neurobiol. Aging 28, 831-844. doi: 10.1016/j.neurobiolaging.2006.04.009

Cristòfol, R., Porquet, D., Corpas, R., Coto-Montes, A., Serret, J., Camins, A., et al. (2012). Neurons from senescence-accelerated SAMP8 mice are protected against frailty by the sirtuin 1 promoting agents melatonin and resveratrol. J. Pineal Res. 52, 271-281. doi: 10.1111/j.1600-079X.2011.00939.x

Day, J. J., and Sweatt, J. D. (2010). DNA methylation and memory formation. Nat. Neurosci. 13, 1319-1323. doi: 10.1038/nn.2666

de Ruijter, A. J. M., van Gennip, A. H., Caron, H. N., Kemp, S., and van Kuilenburg, A. B. P. (2003). Histone deacetylases (HDACs): characterization of the classical HDAC family. Biochem. J. 370(Pt 3), 737-749. doi: 10.1042/BJ20021321

Dwivedi, S., Nagarajan, R., Hanif, K., Siddiqui, H. H., Nath, C., and Shukla, R. (2013). Standardized extract of bacopa monniera attenuates okadaic acid 
induced memory dysfunction in rats: effect on Nrf2 pathway. Evid. Based Complement. Alternat. Med. 2013:294501. doi: 10.1155/2013/294501

Ennaceur, A., and Delacour, J. (1988). A new one-trial test for neurobiological studies of memory in rats. 1. Behavioral data. Behav. Brain Res. 31, 47-59. doi: 10.1016/0166-4328(88)90157-X

Ennaceur, A., and Meliani, K. (1992). A new one-trial test for neurobiological studies of memory in rats. III. Spatial vs. non-spatial working memory. Behav. Brain Res. 51, 83-92. doi: 10.1016/S0166-4328(05)80315-8

Fischer, A., Sananbenesi, F., Wang, X., Dobbin, M., and Tsai, L.-H. (2007). Recovery of learning and memory is associated with chromatin remodelling. Nature 447, 178-182. doi: $10.1038 /$ nature 05772

Fjell, A. M., McEvoy, L., Holland, D., Dale, A. M., and Walhovd, K. B. (2014). What is normal in normal aging? Effects of aging, amyloid and Alzheimer's disease on the cerebral cortex and the hippocampus. Prog. Neurobiol. 117, 20-40. doi: 10.1016/j.pneurobio.2014.02.004

Flax, J. D., and Soloway, P. D. (2011). Methylation on the mind. Nat. Neurosci. 14, 1494-1496. doi: $10.1038 / \mathrm{nn} .2988$

Gräff, J., and Tsai, L.-H. (2013). Histone acetylation: molecular mnemonics on the chromatin. Nat. Rev. Neurosci. 14, 97-111. doi: 10.1038/nrn3427

Griñán-Ferré, C., Pérez-Cáceres, D., Gutiérrez-Zetina, S. M., Camins, A., Palomera-Avalos, V., Ortuño-Sahagún, D., et al. (2015). Environmental enrichment improves behavior, cognition, and brain functional markers in young senescence-accelerated prone mice (SAMP8). Mol. Neurobiol. 53, 24352450. doi: 10.1007/s12035-015-9210-6

Griñán-Ferré, C., Sarroca, S., Ivanova, A., Puigoriol-Illamola, D., Aguado, F., Camins, A., et al. (2016). Epigenetic mechanisms underlying cognitive impairment and Alzheimer disease hallmarks in 5XFAD mice. Aging 8, 664684. doi: 10.18632/aging.100906

Grupe, A., Abraham, R., Li, Y., Rowland, C., Hollingworth, P., Morgan, A., et al. (2007). Evidence for novel susceptibility genes for late-onset Alzheimer's disease from a genome-wide association study of putative functional variants. Hum. Mol. Genet. 16, 865-873. doi: 10.1093/hmg/ddm031

Guan, J. S., Haggarty, S. J., Giacometti, E., Dannenberg, J. H., Joseph, N., Gao, J., et al. (2009). HDAC2 negatively regulates memory formation and synaptic plasticity. Nature 459, 55-60. doi: 10.1038/nature07925

Haettig, J., Stefanko, D. P., Multani, M. L., Figueroa, D. X., McQuown, S. C., and Wood, M. A. (2011). HDAC inhibition modulates hippocampus-dependent long-term memory for object location in a CBP-dependent manner. Learn. Mem. 18, 71-79. doi: 10.1101/lm.1986911

Harold, D., Abraham, R., Hollingworth, P., Sims, R., Gerrish, A., Hamshere, M. L., et al. (2009). Genome-wide association study identifies variants at CLU and PICALM associated with Alzheimer's disease. Nat. Genet. 41, 1088-1093. doi: 10.1038/ng.440

Hernandez, D. G., Nalls, M. A., Gibbs, J. R., Arepalli, S., van, der Brug M, Chong, S., et al. (2011). Distinct DNA methylation changes highly correlated with chronological age in the human brain. Hum. Mol. Genet. 20, 1164-1172. doi: $10.1093 / \mathrm{hmg} / \mathrm{ddq} 561$

Holliday, R. (2006). Epigenetics: a historical overview. Epigenetics 1, 76-80. doi: 10.4161/epi.1.2.2762

Irier, H., Street, R. C., Dave, R., Lin, L., Cai, C., Davis, T. H., et al. (2014). Environmental enrichment modulates 5-hydroxymethylcytosine dynamics in hippocampus. Genomics 104, 376-382. doi: 10.1016/j.ygeno.2014. 08.019

Ito, S., Shen, L., Dai, Q., Wu, S. C., Collins, L. B., Swenberg, J. A., et al. (2011). Tet proteins can convert 5-methylcytosine to 5-formylcytosine and 5-carboxylcytosine. Science 333, 1300-1303. doi: 10.1126/science.1210597

Jurgens, H. A., and Johnson, R. W. (2012). Environmental enrichment attenuates hippocampal neuroinflammation and improves cognitive function during influenza infection. Brain Behav. Immun. 26, 1006-1016. doi: 10.1016/j.bbi.2012.05.015

Kanninen, K., Heikkinen, R., Malm, T., Rolova, T., Kuhmonen, S., Leinonen, H., et al. (2009). Intrahippocampal injection of a lentiviral vector expressing Nrf2 improves spatial learning in a mouse model of Alzheimer's disease. Proc. Natl. Acad. Sci. U.S.A. 106, 16505-16510. doi: 10.1073/pnas.0908397106

Kilgore, M., Miller, C. A., Fass, D. M., Hennig, K. M., Haggarty, S. J., Sweatt, J. D., et al. (2010). Inhibitors of class 1 histone deacetylases reverse contextual memory deficits in a mouse model of Alzheimer's disease. Neuropsychopharmacology 35, 870-880. doi: 10.1038/npp.2009.197
Levenson, J. M., O’Riordan, K. J., Brown, K. D., Trinh, M. A., Molfese, D. L., and Sweatt, J. D. (2004). Regulation of histone acetylation during memory formation in the hippocampus. J. Biol. Chem. 279, 40545-40559. doi: 10.1074/jbc.M402229200

Liu, L., van Groen, T., Kadish, I., and Tollefsbol, T. O. (2009). DNA methylation impacts on learning and memory in aging. Neurobiol. Aging 30, 549-560. doi: 10.1016/j.neurobiolaging.2007.07.020

López-Otín, C., Blasco, M. A., Partridge, L., Serrano, M., and Kroemer, G. (2013). The hallmarks of aging. Cell 153, 1194-1217. doi: 10.1016/j.cell.2013.05.039

Madrigano, J., Baccarelli, A., Mittleman, M. A., Wright, R. O., Sparrow, D., Vokonas, P. S., et al. (2011). Prolonged exposure to particulate pollution, genes associated with glutathione pathways, and DNA methylation in a cohort of older men. Environ. Health Perspect. 119, 977-982. doi: 10.1289/ehp.1002773

Maeda, K., Ohno, T., Igarashi, S., Yoshimura, T., Yamashiro, K., and Sakai, M. (2012). Aldehyde oxidase 1 gene is regulated by Nrf2 pathway. Gene 505, 374-378. doi: 10.1016/j.gene.2012.06.010

Marsit, C. J., Christensen, B. C., Houseman, E. A., Karagas, M. R., Wrensch, M. R., Yeh, R. F., et al. (2009). Epigenetic profiling reveals etiologically distinct patterns of DNA methylation in head and neck squamous cell carcinoma. Carcinogenesis 30, 416-422. doi: 10.1093/carcin/bgp006

Mastroeni, D., Grover, A., Delvaux, E., Whiteside, C., Coleman, P. D., and Rogers, J. (2011). Epigenetic mechanisms in Alzheimer's disease. Neurobiol. Aging 32, 1161-1180. doi: 10.1016/j.neurobiolaging.2010.08.017

Mastroeni, D., McKee, A., Grover, A., Rogers, J., and Coleman, P. D. (2009). Epigenetic differences in cortical neurons from a pair of monozygotic twins discordant for Alzheimer's disease. PLoS ONE 4:e6617. doi: 10.1371/journal.pone.0006617

Mikaelsson, M. A., and Miller, C. A. (2011). The path to epigenetic treatment of memory disorders. Neurobiol. Learn. Mem. 96, 13-18. doi: 10.1016/j.nlm.2011.02.003

Millan, M. J. (2014). The epigenetic dimension of Alzheimer's disease: causal, consequence, or curiosity? Dialog. Clin. Neurosci. 16, 373-393.

Miller, C. A., and Sweatt, J. D. (2007). Covalent modification of DNA regulates memory formation. Neuron 53, 857-869. doi: 10.1016/j.neuron.2007.02.022

Morley, J. E., Armbrecht, H. J., Farr, S. A., and Kumar, V. B. (2012a). The senescence accelerated mouse (SAMP8) as a model for oxidative stress and Alzheimer's disease. Biochim. Biophys. Acta 1822, 650-656. doi: 10.1016/j.bbadis.2011.11.015

Morley, J. E., Farr, S. A., Kumar, V. B., and Armbrecht, H. J. (2012b). The SAMP8 mouse: a model to develop therapeutic interventions for Alzheimer's disease. Curr. Pharm. Des. 18, 1123-1130. doi: 10.2174/138161212799315795

Morris, M. J., Adachi, M., Na, E. S., and Monteggia, L. M. (2014). Selective role for DNMT3a in learning and memory. Neurobiol. Learn. Mem. 115, 30-37. doi: 10.1016/j.nlm.2014.06.005

Morris, R. G. M. (1981). Spatial localization does not requiere the presence of local cues. Learn. Motiv. 12, 239-260. doi: 10.1016/0023-9690(81)90020-5

Neidl, R., Schneider, A., Bousiges, O., Majchrzak, M., Barbelivien, A., de Vasconcelos, A. P., et al. (2016). Late-life environmental enrichment induces acetylation events and nuclear factor $\kappa \mathrm{B}$-dependent regulations in the hippocampus of aged rats showing improved plasticity and learning. J. Neurosci. 36, 4351-4361. doi: 10.1523/JNEUROSCI.3239-15.2016

Oeckinghaus, A., and Ghosh, S. (2009). The NF-kappaB family of transcription factors and its regulation. Cold Spring Harb. Perspect. Biol. 1:a000034. doi: $10.1101 /$ cshperspect.a000034

Pallàs, M. (2012). Senescence-accelerated mice P8: a tool to study brain aging and Alzheimer's disease in a mouse model. ISRN Cell Biol. 2012, 1-12. doi: $10.5402 / 2012 / 917167$

Pallas, M., Camins, A., Smith, M. A., Perry, G., Lee, H., and Casadesus, G. (2008). From aging to Alzheimer's disease: unveiling "the switch" with the senescence-accelerated mouse model (SAMP8). J. Alzheimers Dis. 15, 615-624.

Popa-Wagner, A., Buga, A. M., Adrian-Tica, A., and Valeria Albu, C. (2014). Perfusion deficits, inflammation and aging precipitate depressive behaviour. Biogerontology 15, 439-448. doi: 10.1007/s10522-014.9516-1

Quintanilla, R. A., Orellana, D. I., González-Billault, C., and Maccioni, R. B. (2004). Interleukin-6 induces Alzheimer-type phosphorylation of tau protein by deregulating the cdk5/p35 pathway. Exp. Cell Res. 295, 245-257. doi: 10.1016/j.yexcr.2004.01.002 
Quinti, L., Chopra, V., Rotili, D., Valente, S., Amore, A., Franci, G., et al. (2010). Evaluation of histone deacetylases as drug targets in Huntington's disease models. Study of HDACs in brain tissues from R6/2 and CAG140 knock-in HD mouse models and human patients and in a neuronal HD cell model. PLoS Curr. 2:RRN1172. doi: 10.1371/currents.RRN1172

Richardson, B. (2003). Impact of aging on DNA methylation. Ageing Res. Rev. 2, 245-261. doi: 10.1016/S1568-1637(03)00010-2

Ricobaraza, A., Cuadrado-Tejedor, M., Pérez-Mediavilla, A., Frechilla, D., Del Río, J., and García-Osta, A. (2009). Phenylbutyrate ameliorates cognitive deficit and reduces tau pathology in an Alzheimer's disease mouse model. Neuropsychopharmacology 34, 1721-1732. doi: 10.1038/npp.2008.229

Snow, W. M., Pahlavan, P. S., Djordjevic, J., McAllister, D., Platt, E. E., Alashmali, S., et al. (2015). Morris water maze training in mice elevates hippocampal levels of transcription factors nuclear factor (erythroid-derived 2)-like 2 and nuclear factor kappa B p65. Front. Mol. Neurosci. 8:70. doi: 10.3389/fnmol.2015.00070

Spiegel, A. M., Sewal, A. S., and Rapp, P. R. (2014). Epigenetic contributions to cognitive aging: disentangling mindspan and lifespan. Learn. Mem. 21, 569-574. doi: 10.1101/lm.033506.113

Stefanko, D. P., Barrett, R. M., Ly, A. R., Reolon, G. K., and Wood, M. A. (2009). Modulation of long-term memory for object recognition via HDAC inhibition. Proc. Natl. Acad. Sci. U.S.A. 106, 9447-9452. doi: 10.1073/pnas.0903964106

Sureda, F. X., Gutierrez-Cuesta, J., Romeu, M., Mulero, M., Canudas, A. M., Camins, A., et al. (2006). Changes in oxidative stress parameters and neurodegeneration markers in the brain of the senescence-accelerated mice SAMP-8. Exp. Gerontol. 41, 360-367. doi: 10.1016/j.exger.2006.01.015

Takeda, T. (2009). Senescence-accelerated mouse (SAM) with special references to neurodegeneration models, SAMP8 and SAMP10 mice. Neurochem. Res. 34, 639-659. doi: 10.1007/s11064-009-9922-y

Weber, M., Hellmann, I., Stadler, M. B., Ramos, L., Pääbo, S., Rebhan, M., et al. (2007). Distribution, silencing potential and evolutionary impact of promoter DNA methylation in the human genome. Nat. Genet. 39, 457-466. doi: $10.1038 /$ ng 1990
Williamson, L. L., Chao, A., and Bilbo, S. D. (2012). Environmental enrichment alters glial antigen expression and neuroimmune function in the adult rat hippocampus. Brain Behav. Immun. 26, 500-510. doi: 10.1016/j.bbi.2012. 01.003

Wu, S. C., and Zhang, Y. (2010). Active DNA demethylation: many roads lead to Rome. Nat. Rev. Mol. Cell Biol. 11, 607-620. doi: 10.1038/ nrm2950

Yuan, Z., Wang, M., Yan, B., Gu, P., Jiang, X., Yang, X., et al. (2012). An enriched environment improves cognitive performance in mice from the senescence-accelerated prone mouse 8 strain: role of upregulated neurotrophic factor expression in the hippocampus. Neural Regen. Res. 7, 1797-1804. doi: 10.3969/j.issn.1673-5374.2012.23.006

Zaheer, S., Thangavel, R., Wu, Y., Khan, M. M., Kempuraj, D., and Zaheer, A. (2013). Enhanced expression of glia maturation factor correlates with glial activation in the brain of triple transgenic Alzheimer's disease mice. Neurochem. Res. 38, 218-225. doi: 10.1007/s11064-012-0913-Z

Zhao, Z., Fan, L., Fortress, A. M., Boulware, M. I., and Frick, K. M. (2012). Hippocampal histone acetylation regulates object recognition and the estradiolinduced enhancement of object recognition. J. Neurosci. 32, 2344-2351. doi: 10.1523/JNEUROSCI.5819-11.2012

Conflict of Interest Statement: The authors declare that the research was conducted in the absence of any commercial or financial relationships that could be construed as a potential conflict of interest.

Copyright (C) 2016 Griñan-Ferré, Puigoriol-Illamola, Palomera-Ávalos, PérezCáceres, Companys-Alemany, Camins, Ortuño-Sahagún, Rodrigo and Pallàs. This is an open-access article distributed under the terms of the Creative Commons Attribution License (CC BY). The use, distribution or reproduction in other forums is permitted, provided the original author(s) or licensor are credited and that the original publication in this journal is cited, in accordance with accepted academic practice. No use, distribution or reproduction is permitted which does not comply with these terms. 